\title{
High Resolution Distributed Optical Fiber Sensing Using Time-Expanded Phase-Sensitive Reflectometry
}

\author{
María R. Fernández-Ruiz ${ }^{1 *}$, Miguel Soriano-Amat ${ }^{1}$, Hugo F. Martins ${ }^{2}$, Vicente Durán ${ }^{3}$, \\ Sonia Martin-Lopez ${ }^{1}$ and Miguel Gonzalez-Herraez ${ }^{1}$
}

${ }^{1}$ Departamento de Electrónica, Escuela Politécnica Superior, Universidad de Alcalá, Alcalá de Henares, Spain, ${ }^{2}$ Instituto de Óptica "Daza de Valdés", IO-CSIC, Madrid, Spain, "3Institute of New Imaging Technologies, University Jaume I, Castellón, Spain

We have demonstrated a novel scheme for distributed optical fiber sensing based on the use of a dual frequency comb, which enables the development of a high-resolution (in the $\mathrm{cm}$ range) distributed sensor with significantly relaxed electronic requirements compared with previous schemes. This approach offers a promising solution for real time structure monitoring in a variety of fields, including transportation, manufacturing or mechatronics. In this work, we review the principle of operation of the technique, recent advances to improve its performance and different experimental tests.

OPEN ACCESS

Edited by:

Daniele Tosi,

Nazarbayev University, Kazakhstan

Reviewed by:

Riqing $L V$,

Northeastern University, China

${ }^{*}$ Correspondence:

María R. Fernández-Ruiz rosario.fernandezr@uah.es

Specialty section: This article was submitted to Physical Sensors,

a section of the journal

Frontiers in Sensors

Received: 29 October 2021 Accepted: 13 December 2021

Published: 05 January 2022

Citation:

Fernández-Ruiz MR, Soriano-Amat M, Martins HF, Durán V, Martin-Lopez S and Gonzalez-Herraez M (2022) High Resolution Distributed Optical Fiber Sensing Using Time-Expanded Phase-

Sensitive Reflectometry.

Front. Sens. 2:804556.

doi: 10.3389/fsens.2021.804556
Keywords: fiber optics, Rayleigh scattering, dual comb spectroscopy, distributed acoustic sensing, structure health monitoring

\section{INTRODUCTION}

Distributed optical fiber sensors (DOFS) have gained a great deal of attention for their appealing advantages as the high number of available sensing points with minimal intrusiveness, their lightweight and simplicity (Lu et al., 2019; Lindsey et al., 2019). In particular, DOFS based on phase sensitive OTDR (TOTDR) have demonstrated their ability to perform real time monitoring of structures with sampling frequencies reaching the acoustic range (i.e., up to the $\mathrm{kHz}$ regime) (Wang et al., 2016; Zhang et al., 2016). However, they are typically limited to spatial resolutions of several meters, making them only cost-efficient in long-range $(>1 \mathrm{~km})$ applications. Finer spatial resolution has been attempted by the modulation of the probe pulse, e.g., using linear frequency modulation ( $\mathrm{Lu}$ et al., 2017) or phase-shift keying (PSK) modulation formats (Martins et al., 2016), in combination with matched filters digitally applied on the coherently detected traced. By use of these strategies, centimeter-scale resolutions have been attained, at the cost of severely increasing the photodetection and acquisition bandwidths up to several $\mathrm{GHz}$ (e.g., $5 \mathrm{GHz}$ are required for resolutions of $2 \mathrm{~cm}$ ). This fact not only increases the system cost, but also implies heavy computational load due to the massive amount of data acquired.

Recently, a novel interrogation technique for ФOTDR has been formalized and demonstrated. It is based on the use of a dual frequency comb (DFC) as the ones typically employed in spectroscopy (Coddington et al., 2016). A DFC consists in a pair of nearly identical, mutually coherent frequency combs having a slight mismatch in the comb line space. In the newly developed interrogation methodology, one comb is launched into the fiber under test and the Rayleigh backscattered trace is beaten with the second comb, which is used as a local oscillator (LO). Upon photodetection, the mismatch in the comb line spacing induces a multi-heterodyne process, downconverting the optical traces to the radio-frequency (RF) domain. This interrogation process is tremendously efficient in terms of detection and acquisition bandwidths, since optical probe combs with bandwidths relatively wide (e.g., several $\mathrm{GHz}$ ), corresponding to fine spectral resolutions (e.g., in the $\mathrm{cm}$ scale), are 
compressed down to the $\mathrm{MHz}$ regime in detection. This spectral compression is equivalent to an expansion of the detected traces in time domain. For this reason, the technique has been called time-expanded (TE-)ФOTDR (Soriano-Amat et al., 2021b). The high spatial resolution attained, along with the capabilities of performing real time operation even in relatively long fibers (hundreds of meters) with a simple and potentially costeffective setup, position this sensing method in a unique place, as its performance is not overlapped by any other distributed optical fiber sensing technology. In this way, TE- ФOTDR arises as an excellent solution for structural health monitoring of moderate-size structures such as vehicles, buildings, wing turbines, etc.

This Review article is organized as follows: In Theoretical Trade-Offs of TE- $\Phi O T D R$, we revise the performance limits of TE- OTDR, providing details on the reported techniques to beat the trade-offs imposed by the use of a dual comb. Namely, a codification technique to improve the sensitivity is described in Coding Strategies; and the use of a quasi-integer ratio dual comb to beat the trade-off between range, resolution and sampling frequency is described in Quasi-Integer Ratio Operation. Experimental Results sums up the works including experimental results that validate the different techniques explained in Theoretical Trade-Offs of TE- $\Phi O T D R$. Finally, Discussion offers a general discussion comparing the performance of TE-ФOTDR with previous optical fiber interrogation techniques.

\section{THEORETICAL TRADE-OFFS OF TE-ФOTDR}

As previously introduced, TE- OOTDR involves the use of a frequency comb to probe the fiber under test and another comb employed as a LO.

The probe arm of the system works exactly as in a traditional ФOTDR, maintaining all their trade-offs. Namely, a train of highly coherent optical pulses is launched into the fiber under test. The repetition rate of the pulse train $\left(f_{R}\right)$ limits the range of the sensor, since the trace generated by one pulse must reach the front end of the fiber before sending another pulse. In particular, the maximum range is $L_{\max }=c / 2 n f_{R}$, with $c$ the speed of light in a vacuum and $n$ the effective refractive index of the fiber. The pulse width of the pulses $\left(\tau_{p}\right)$ limits the sensor resolution $(\Delta s)$ (defined as the minimum distance between two resolvable points) in the case of transform-limited pulses (where $\tau_{p} \sim 1 / B_{o}$, with $B_{o}$ the optical bandwidth), i.e., $\Delta s=\tau_{p} c /(2 n)$.

The optical traces backscattered in the fiber interfere with the LO comb in the photodetector. The process is equivalent to an asynchronous optical sampling (ASOPS) (Bartels et al., 2007), where the response of the fiber is sampled by the probe comb, and the resulting signal is asynchronously sampled by the LO comb. Upon interference of the pair of optical combs in the photodetector, beating notes between all the comb lines appear in the electrical domain, leading to two RF combs per optical line spacing. To avoid aliasing in this process, it is important that the number of lines is bounded $(N)$ and that the maximum offset between pairs of teeth is $N \cdot \delta f<f_{R} / 2$. For this reason, when working with DFCs, the comb spectral envelope must be well limited, ideally being rectangular-like. That means that, in the transform limited case, the optical probe pulses are sinc-like shaped. The sinc sidelobes generally degrade the effective spatial resolution of the sensor in the presence of neighboring perturbations. From the different RF combs that appears in the electrical domain, the first one, located between DC and $f_{R} / 2$ (also known as first Nyquist zone, see Figure 1A), contains the beating notes between neighboring lines of the optical dual comb. The line space of this comb is equal to the line space offset of the dual comb, $\delta f$, and its bandwidth is $B_{R F}=B_{o} \delta f / f_{R}$, being the ratio $f_{R} / \delta f$ defined as the compression factor $(C F)$. By filtering the comb located in the first Nyquist zone, we obtain the optical traces expanded from $L \leq L_{\max }$ to $L \cdot C F$ in time domain (Figure 1A). It has been demonstrated that the expansion process affects the traces SNR similarly to an averaging process, i. e., the resulting traces has an improvement of SNR of $\sqrt{ } C F$ (Soriano-Amat et al., 2021b).

To date, in the bibliography related to TE- OTDR, the DFC has been generated by an electro-optic (EO) setup (Figure 1B). In particular, the combs have been computationally designed and subsequently generated by a 2-channel arbitrary waveform generator (AWG). The electrical combs drive two MachZehnder modulators (MZM) which modulate a highly coherent continuous wave (CW). This configuration provides high flexibility to generate the combs, in terms of bandwidth, line space, and offset between the two comb line spaces. However, the use of an AWG increases the system cost. Alternative means for the electrical comb generation are to be tested aimed at simplifying the generation scheme, such as the use of field programmable gate arrays (FPGAs) (Fdil et al., 2019).

In the remainder of this section, we will review several strategies employed in TE- OOTDR for beating the trade-offs in SNR and sensing range and/or sampling frequency.

\section{Coding Strategies}

An optical frequency comb with a flat spectral phase corresponds to a train of pulses in time domain, where the comb line space fixes the pulse repetition rate and the pulse shape corresponds to the inverse Fourier transform of the comb spectral envelope. In TE- OOTDR, the number of comb lines, $N$, imposes the effective number of sensing points provided by the fiber. Namely, $N=L / \Delta s=B_{o} / f_{R}$. Hence, a high number of comb lines is a desired goal for distributed sensing. This implies that the timedomain transform-limited pulse train has very low duty cycle, i.e., very narrow pulses with a very long period. For the probe signal to have sufficiently high energy, it is necessary that the pulses have a high peak power. However, high-peak-power pulses could induce nonlinearities in the drivers and EO modulators, or along the fiber under test. To increase the SNR of the traces while avoiding the onset of nonlinear effects along the circuit, several coding strategies have been tested.

First, the phase of each spectral comb line was modulated with a random value between 0 and $2 \pi$ (Soriano-Amat et al., 2021b). The phases of the probe comb and LO comb were encoded using the same phase profile. By using this strategy, the time domain 

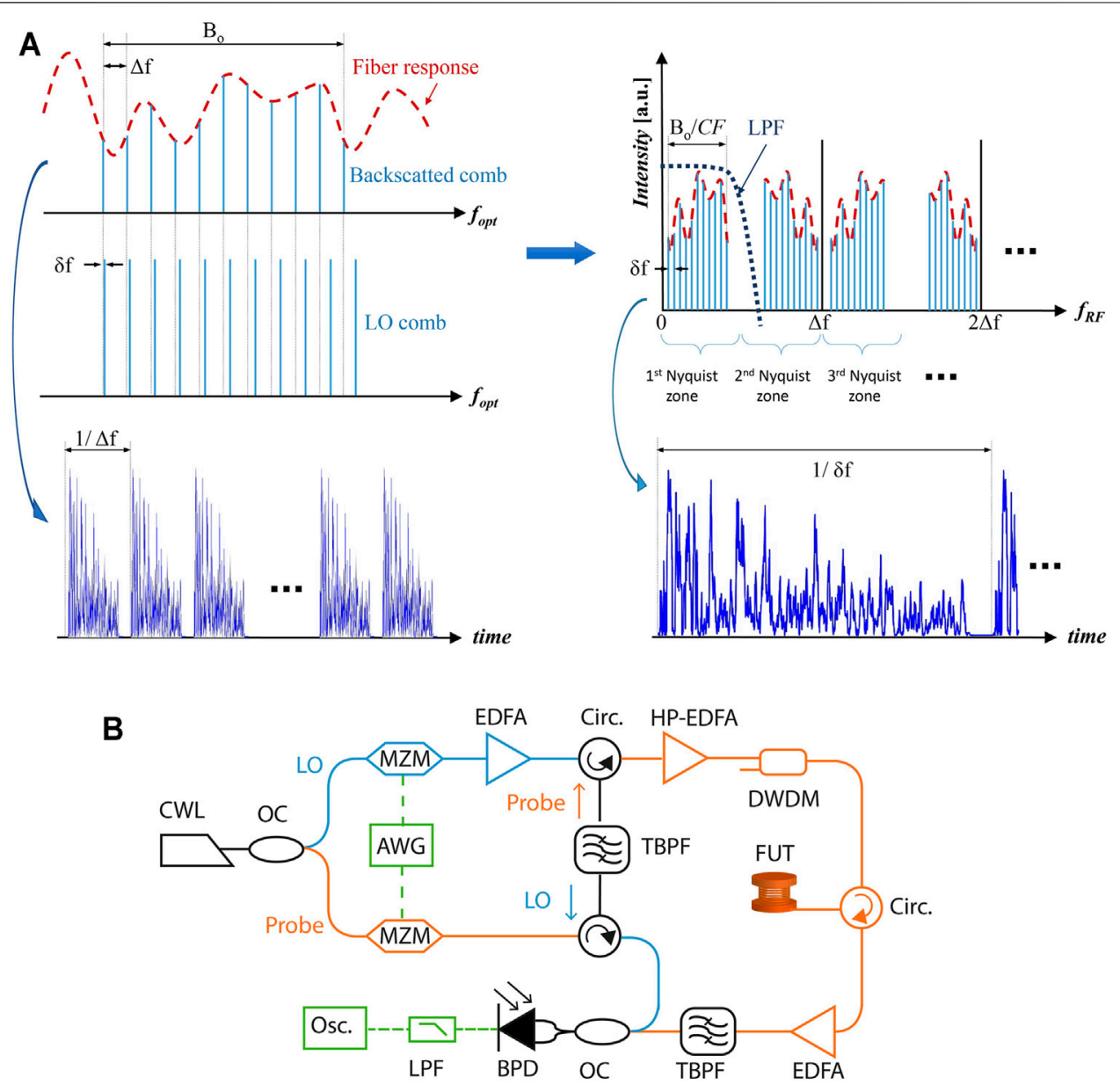

FIGURE 1 | (A) Principle of operation of TE-ФOTDR. Upon interference of the backscattered and local oscillator (LO) optical combs, pairs of RF combs appear within each line space interval in the electrical domain. By filtering the comb located in the first Nyquist zone, we obtain in time domain a time-expanded trace providing information on the fiber response. LPF: Low pass filter. Comb parameters are defined in the manuscript. (B) Experimental setup of a TE- $\Phi O T D R$ scheme. Acronyms: CWL: continuous wave laser; OC: optical coupler; LO: local oscillator; MZM: Mach-Zehnder modulator; AWG: arbitrary waveform generator; EDFA: erbium-doped fiber amplifier; TBPF: Tunable band pass filter; Circ: circulator; HP-EDFA: high-power EDFA; DWDM: dense wavelength division multiplexer; FUT: fiber under test; BPD: balanced photodetector; LPF: low-pass filter; Osc: Oscilloscope.

pulses transform into a speckle-like shape covering the full period. Thus, the peak-to-average power ratio (PAPR) of the probe and LO signals can be significantly reduced with respect to the transform-limited case [in almost two orders of magnitude in (Soriano-Amat et al., 2021b)]. The PAPR reduction provides a proportional increase in the SNR of the obtained traces. The advantage of using the same phase profile in both the probe and LO combs is that, upon detection, the RF comb at the first Nyquist zone is automatically decoded, with no need for using digital matched filters after detection. Hence, the time-expanded response of the optical fiber is directly obtained by simply filtering in that low-frequency comb.

To further increase the SNR of the traces, a more sophisticated coding strategy has been also tested in (Soriano-Amat et al., 2021c). In particular, the spectral phase of rectangular-envelope frequency combs is modulated with a quadratic profile. The quadratic coefficient is selected so that the pulses suffer a frequency-to-time mapping (Torres-Company et al., 2011) and hence, their envelope gets rectangular-like with a width similar to the pulse train period. In this way, the modulus of the timedomain signal becomes nearly continuous wave, reducing the PAPR to nearly the optimal situation $(\mathrm{PAPR}=1)$. In practice, $\mathrm{PAPR}=1$ is not attained, due to the residual tails of the resulting time domain pulses. Nevertheless, a PAPR of 1.58 has been reached in the experimental demonstration reported in (Soriano-Amat et al., 2021c). In that work, the SNR of the traces increased in $8 \mathrm{~dB}$ with respect to the case where random spectral phase was employed, and a theoretical improvement of $25 \mathrm{~dB}$ was estimated with respect to the transform-limited case.

\section{Quasi-Integer Ratio Operation}

The use of DFCs imposes a stringent limitation in sensing range and/or sensing sampling frequency, $f_{S}=\delta f$, in TE-ФOTDR configurations. The reason is that, to avoid aliasing in the down-conversion process, the offset between comb line spaces 

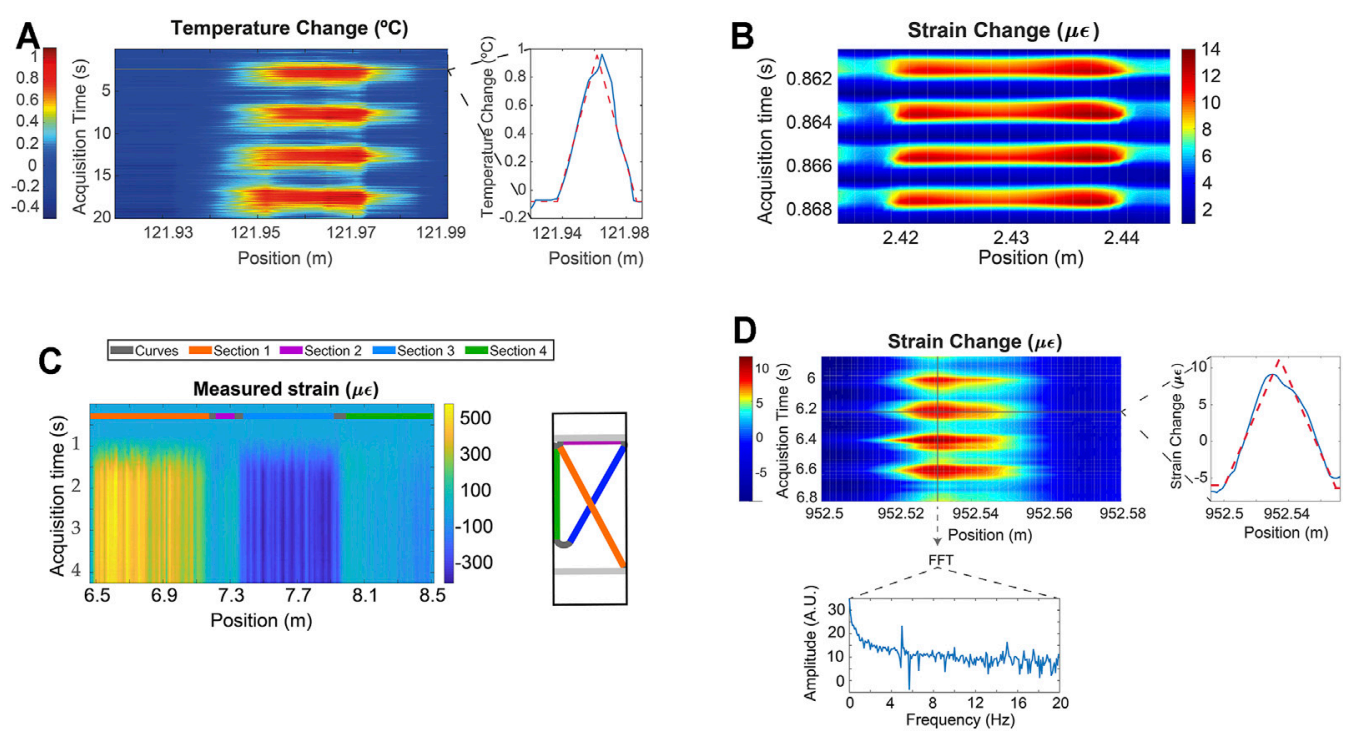

FIGURE 2 | Experimental results obtained from the TE$20 \mathrm{~Hz}$ and range of $200 \mathrm{~m}$. The inset shows the spatial-domain shape of the detected perturbation, which corresponds to the convolution of a square-shaped perturbation profile and the $2 \mathrm{~cm}$ resolution. (B) Measurement of strain perturbation with resolution of $2 \mathrm{~cm}$, sampling frequency of $2 \mathrm{kHz}$ and range of $10 \mathrm{~m}$, using a specially designed quadratic spectral phase modulation. (C) Torsion of a model of wing of an unmanned aerial vehicle. The fiber cable was glued on the wing using the topology shown in the inset of figure. (D) Measurement of a periodic strain perturbation with resolution of $4 \mathrm{~cm}$, sampling frequency of $40 \mathrm{~Hz}$ and range of $1 \mathrm{~km}$, using the quasi-integer ratio dual comb configuration. Inset at the right shows the spatial-domain detected profile and inset at the bottom shows the fast Fourier transform (FFT) measured at the marked position of the strain map.

has to be limited to $\delta f<f_{R}^{2} /\left(2 B_{o}\right)$ (as described in Theoretical Trade-Offs of TE- $\Phi O T D R)$. By writing this expression in terms of the sensor performance, we have that

$$
f_{S}<\frac{c}{4 n} \cdot \frac{\Delta s}{L_{\max }^{2}}
$$

Hence, if a high number of sensing points is pursued (e.g., 10,000 points), the sensing bandwidth is necessarily reduced (e.g., $25 \mathrm{~Hz}$, considering $2 \mathrm{~cm}$ resolution). On the other hand, fine resolution and high sampling frequency (e.g., $2 \mathrm{~cm}$ resolution and $500 \mathrm{~Hz}$ ) is only reachable in short fibres (e.g., $<45 \mathrm{~m}$ ).

This severe condition can be relaxed if another concept from the dual comb spectroscopy field is implemented, namely, the use of a quasi-integer dual comb (Hébert et al., 2014). This concept has been briefly investigated in (Soriano-Amat et al., 2021b) for extending the range of operation of TE-ФOTDR. The modification to the original TE- OOTDR method consists in using two frequency combs with repetition rates that are quasi-integer multiples. For example, if the line space of probing comb was $f_{R}$, the linespace of the LO comb would be $f_{R}^{\prime}=M \cdot f_{R}+\delta f$. Here, in each Nyquist zone, a comb with $N / M$ lines appears, and the complete probing comb can be reconstructed by filtering in a band equal to $M \cdot f_{R}$ and reorganizing the lines. A degree of freedom is thus added to the previously proposed method, since in this case, it must be accomplished that $\delta f<M \cdot f_{R}^{2} /\left(2 B_{o}\right)$, which permits increasing the range and/or the sampling frequency for a particular target resolution. This relaxation in the trade-off imposed by the dual comb comes at the cost of an $M$-fold increase in the detection and digitization bandwidth, and a rise of some processing complexity (still low in comparison with normal coding strategies).

\section{EXPERIMENTAL RESULTS}

Even through TEpossible to find several interesting experimental demonstrations of the capabilities of the technique. Namely, there are experimental tests of strain measurements, temperature monitoring, and eminently practical measurements such as monitoring of deformations in the wing of an unmanned aerial vehicle (UAV). In Figure 2, we show some of the experimental results obtained from the technique. In particular, in Figure $\mathbf{2 A}$, periodical temperature variations with a resolution of $2 \mathrm{~cm}$ and sampling rate of $20 \mathrm{~Hz}$ over a fiber of about $200 \mathrm{~m}$ have been detected and reported in (SorianoAmat et al., 2021b). For this experimental demonstration, a random spectral phase modulation was employed in the dual comb. The obtained traces had an SNR of about $20 \mathrm{~dB}$. Such a high SNR is owed to the coding strategy and the SNR enhancement attained by the time-expansion of the traces (the CF in this case was 12,500). Later in (Soriano-Amat et al., 2021c), the SNR improvement given by the use of a specially designed quadratic spectral phase was evaluated. An SNR improvement of $8 \mathrm{~dB}$ was attained by following this strategy, as compared with the random spectral coding. In that work, strain measurements with 
resolution of $2 \mathrm{~cm}$, and sampling rate of $2 \mathrm{kHz}$ were performed over a shorter fiber range of $3.56 \mathrm{~m}$. Results are shown in Figure 2B. The technique has been evaluated for monitoring the bonding, torsion and vibration of a model of wing of an UAV in (Soriano-Amat et al., 2021a). For this purpose, an appropriate topology of fiber cable was glued on the wing (shown in the inset of Figure 2C) in order to measure any 2D perturbation. The strain map obtained from the torsion of the wing is plotted in Figure 2C. Finally, an example of QIR operation was tested in (Soriano-Amat et al., 2021b), using a value of $M=50$. This has enabled to measure strain perturbations with a resolution of $4 \mathrm{~cm}$, sampling rate of $40 \mathrm{~Hz}$ over a fiber range of $1 \mathrm{~km}$ (totaling 25,000 sensing points). The strain map obtained in this case is shown in Figure 2D. The SNR of the trace in this case is $9.3 \mathrm{~dB}$ due to the fact that the $C F$ is reduced $M$ fold and the employed phase coding was not completely random. Instead, blocks of $M$ consecutive lines had the same phase to avoid the need for post-processing in detection.

\section{DISCUSSION}

TE-ФOTDR is a very recent interrogation method for Rayleigh-based DOFS. Even so, it has been demonstrated that TE-ФOTDR offers sensing performance not achieved by any other distributed optical sensing technique. Compared with other time-domain Rayleigh based sensors (i.e., ФOTDR), it provides high spatial resolution with extraordinarily relaxed requirements in terms of detection and acquisition bandwidths and no need for post processing algorithms such as matched filters or others. However, implementations to date translate the EO complexity to the comb generation stage. Even if possibilities for cheap comb generators exist in the market (e.g., based on the use of FPGAs), their application in a TE- OOTDR scheme is still missing. On the other hand, compared with frequency domain Rayleigh-based sensors, the attainable spatial resolution is

\section{REFERENCES}

Bartels, A., Cerna, R., Kistner, C., Thoma, A., Hudert, F., Janke, C., et al. (2007). Ultrafast Time-Domain Spectroscopy Based on High-Speed Asynchronous Optical Sampling. Rev. Scientific Instr. 78 (3), 035107-035108. doi:10.1063/ 1.2714048

Coddington, I., Newbury, N., and Swann, W. (2016). Dual-Comb Spectroscopy. Optica. 3 (4), 414-425. doi:10.1364/optica.3.000414

Fdil, K., Michaud-Belleau, V., Hébert, N. B., Guay, P., Fleisher, A. J., Deschênes, J.D., et al. (2019). Dual Electro-Optic Frequency Comb Spectroscopy Using Pseudo-random Modulation. Opt. Lett. 44 (17), 4415-4418. doi:10.1364/ ol.44.004415

Hébert, N. B., Boudreau, S., Genest, J., and Deschênes, J.-D. (2014). Coherent DualComb Interferometry with Quasi-Integer-Ratio Repetition Rates. Opt. Express 22 (23), 29152-29160. doi:10.1364/oe.22.029152

Lindsey, N. J., Dawe, T. C., and Ajo-Franklin, J. B. (2019). Illuminating Seafloor Faults and Ocean Dynamics with Dark Fiber Distributed Acoustic Sensing. Science 366 (6469), 1103-1107. doi:10.1126/science.aay5881

Lu, B., Pan, Z., Wang, Z., Zheng, H., Ye, Q., Qu, R., et al. (2017). High Spatial Resolution Phase-Sensitive Optical Time Domain Reflectometer with a comparable, but TE-ФOTDR permits real time monitoring over longer ranges with higher sampling rate, especially if QIR dual combs are employed. The interested reader can find a table comparing the performance of relative optical fiber sensing techniques in (Soriano-Amat et al., 2021b). Note that an extensive review of optical fiber sensing techniques was out-of-scope of this work.

TE- -OTDR arises as an ideal distributed sensing technique in those cases in which real time monitoring of a high number of sensing points (e.g., in the $10^{4}$ range) and fine spatial resolution (centimeter scale) are required. Examples of such applications are the structure health monitoring of transportation vehicles, buildings, manufacturing technology and any kind of moderate-size structures.

\section{AUTHOR CONTRIBUTIONS}

Original draft preparation: MF-R. Artwork preparation and experimental demonstration of referred works: MS-A. Data post-processing of referred works: MS-A, HM. Review of manuscript and supervision: MF-R, VD, SM-L, MG-H.

\section{FUNDING}

Comunidad de Madrid and FEDER Program (grant SINFOTON2-CM: P2018/NMT-4326), Generalitat Valenciana (grant PROMETEO/2020/029), the European Research Council (OCEAN-DAS: ERC-2019-POC-875302), the Spanish Government (projects RTI 2018-097957-B-C31, RTI 2018-097957-B-C32, and RTI 2018-097957-B-C33), Universitat Jaume I (project UJI-B2019-45) and Universidad de Alcalá (project CCG20/IA-028). MS-A, HM, VD, and MFR. acknowledge financial support from the Spanish MICINN under contracts no. PRE-2019-087444, IJCI-2017-33856, RYC2017-23668, and IJC2018-035684-I, respectively.

Frequency-Swept Pulse. Opt. Lett. 42 (3), 391-394. doi:10.1364/ ol.42.000391

Lu, P., Lalam, N., Badar, M., Liu, B., Chorpening, B. T., Buric, M. P., et al. (2019). Distributed Optical Fiber Sensing: Review and Perspective. Appl. Phys. Rev. 6 (4), 041302. doi:10.1063/1.5113955

Martins, H. F., Shi, K., Thomsen, B. C., Martin-Lopez, S., Gonzalez-Herraez, M., and Savory, S. J. (2016). Real Time Dynamic Strain Monitoring of Optical Links Using the Backreflection of Live PSK Data. Opt. Express 24 (19), 22303-22317. doi:10.1364/OE.24.022303

Soriano-Amat, M., Fragas-Sánchez, D., Martins, H. F., Vallespín-Fontcuberta, D., Preciado-Garbayo, J., Martin-Lopez, S., et al. (2021a). Monitoring of a Highly Flexible Aircraft Model Wing Using Time-Expanded Phase-Sensitive Otdr. Sensors 21 (3766), 1-10. doi:10.3390/s21113766

Soriano-Amat, M., Martins, H. F., Durán, V., Costa, L., Martin-Lopez, S., Gonzalez-Herraez, M., et al. (2021b). Time-Expanded Phase-Sensitive Optical Time-Domain Reflectometry. Light Sci. Appl. 10 (51), 1-12. doi:10.1038/s41377-021-00490-0

Soriano-Amat, M., Martins, H. F., Durán, V., Martin-Lopez, S., Gonzalez-Herraez, M., and Fernández-Ruiz, M. R. (2021c). Quadratic Phase Coding for SNR Improvement in Time-Expanded Phase-Sensitive OTDR. Opt. Lett. 46 (17), 4406-4409. doi:10.1364/ol.432350 
Torres-Company, V., Leaird, D. E., and Weiner, A. M. (2011). Dispersion Requirements in Coherent Frequency-To-Time Mapping. Opt. Express 19 (24), 24718-24729. doi:10.1364/oe.19.024718

Wang, Z., Zhang, L., Wang, S., Xue, N., Peng, F., Fan, M., et al. (2016). Coherent $\Phi-O T D R$ Based on I/Q Demodulation and Homodyne Detection. Opt. Express 24 (2), 853-858. doi:10.1364/OE.24.000853

Zhang, J., Zhu, T., Zhou, H., Huang, S., Liu, M., and Huang, W. (2016). High Spatial Resolution Distributed Fiber System for Multi-Parameter Sensing Based on Modulated Pulses. Opt. Express 24 (24), 27482. doi:10.1364/OE.24.027482

Conflict of Interest: The authors declare that the research was conducted in the absence of any commercial or financial relationships that could be construed as a potential conflict of interest.
Publisher's Note: All claims expressed in this article are solely those of the authors and do not necessarily represent those of their affiliated organizations, or those of the publisher, the editors and the reviewers. Any product that may be evaluated in this article, or claim that may be made by its manufacturer, is not guaranteed or endorsed by the publisher.

Copyright $\odot 2022$ Fernández-Ruiz, Soriano-Amat, Martins, Durán, Martin-Lopez and Gonzalez-Herraez. This is an open-access article distributed under the terms of the Creative Commons Attribution License (CC BY). The use, distribution or reproduction in other forums is permitted, provided the original author(s) and the copyright owner(s) are credited and that the original publication in this journal is cited, in accordance with accepted academic practice. No use, distribution or reproduction is permitted which does not comply with these terms. 